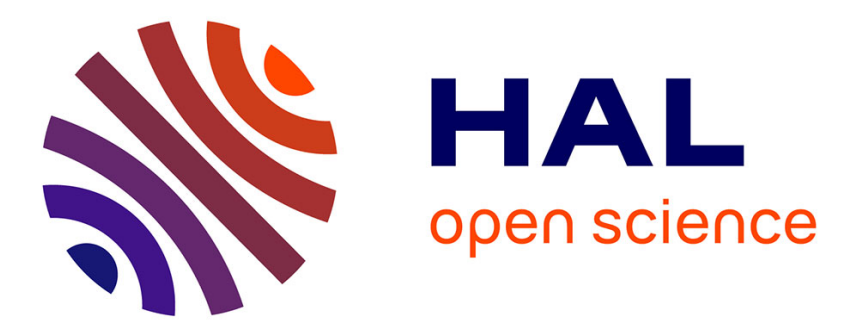

\title{
Effect of Plant Interaction on Wind-Induced Crop Motion
}

\author{
Olivier Doaré, Bruno B. Moulia, Emmanuel de Langre
}

\section{To cite this version:}

Olivier Doaré, Bruno B. Moulia, Emmanuel de Langre. Effect of Plant Interaction on Wind-Induced Crop Motion. Journal of Biomechanical Engineering, 2004, 126 (2), pp.146-151. 10.1115/1.1688773 . hal-00830695

\section{HAL Id: hal-00830695 \\ https://hal-ensta-paris.archives-ouvertes.fr/hal-00830695}

Submitted on 19 Jan 2018

HAL is a multi-disciplinary open access archive for the deposit and dissemination of scientific research documents, whether they are published or not. The documents may come from teaching and research institutions in France or abroad, or from public or private research centers.
L'archive ouverte pluridisciplinaire HAL, est destinée au dépôt et à la diffusion de documents scientifiques de niveau recherche, publiés ou non, émanant des établissements d'enseignement et de recherche français ou étrangers, des laboratoires publics ou privés. 


\title{
Effect of Plant Interaction on Wind-Induced Crop Motion
}

\author{
O. Doaré Département de mécanique, LadHyX, CNRS-Ecole Polytechnique, 91128, Palaiseau, France
}

\section{B. Moulia UEPF-INRA, 86600 Lusignan, FRANCE}

E. de Langre Département de meécanique, LadHyX, CNRS-Ecole Polytechnique, 91128 Palaiseau, FRANCE

Plant motion due to wind affects plant growth, a phenomenon called thigmomorphogen-esis. Despite intensive studies of the turbulence over plant canopies, the study of plant motion induced by wind has often been limited to individual trees or cereal plants. Few models of global canopy motions are available. Moreover the numerical analysis of mod-els that are based on individual stems becomes time consuming when dealing with crops. A model of motion within the canopies is proposed here using a wave propagation equa-tion within a homogenized continuous medium, and a forcing function representing tur-bulent gusts advected over the canopy. This model is derived from a discrete model of a set of plant shoots represented as individual oscillators, including elastic contacts between shoots. Such contacts induce nonlinearities into the wave equation. A new experi-mental method to measure stem dynamical properties and elastic collision properties is presented with an illustration on alfalfa stems. Results obtained modeling plant motions in an alfalfa crop are presented.

\section{Introduction}

Wind flow over plant canopies is turbulent (see [1] for a recent review) and results in oscillatory motions of individual plants [2,3]. These motions involve bending-and sometimes torsional-strains within the stem of the plant. When these strains are high enough, failure may appear within the plant tissues. Lodging appears, and may reduce the agricultural yield [4]. However, even when the wind load does not induce failures and lodging, plant motion can influence the growth and the quality of the crop. Plants have been shown to be sensitive to mechanical perturbations and to respond to them by reducing the growth in height and increasing the growth in girth, a phenomenon called thigmomorphogenesis $[5,6]$. Plant motion may also influence the vertical convective air fluxes between the canopy and the atmosphere with possible consequences to $\mathrm{CO}_{2}$ transport and canopy photosynthesis, water losses and even pathogen development $[2,7]$.

Several authors have studied the oscillatory behavior of isolated individual stems in free bending, representing the stem as a simple mono- or bi-dimensional oscillating rod $[2,3,4,8]$. The resulting resonance frequency in the fundamental mode has been shown to match peaks in the power spectrum of plant motion in canopies. However to our knowledge only one attempt has been made to model the motions of a set of plants within a canopy exposed to wind. This model $[7,9]$ describes a wheat canopy as a discrete set of oscillating rods whose motion represents the oscillation of a set of plant stems along a row. It includes the dynamics of the vibrations of successive stems when exposed to a moving force representing the motion of turbulent wind gusts, but neglects the mechanical interactions between neighboring plants. In this paper, our aim is to describe the influence of elastic collisions between neighboring plants on the dynamics of the crop canopy. For this purpose, we develop a simple model for the behavior of individual plants and wind profiles and introduce elastic contact between stems. Then, an improved model based on a wave equation for an equivalent continuous medium representing the canopy is derived. Simple models of wind load are chosen from the literature. These models are then applied to a canopy of alfalfa.

*Present address: UME/ENSTA, Chemin de la Hunière, 91761 Palaiseau Cedex, FRANCE.

\section{The Crop Canopy Model}

2.1 Discrete Crop Canopy Model Including Plant-to-Plant Contacts. A typical stem usually has two directions in which free oscillations are planar (Flesh [10] and Farquhar [4]). For the sake of simplicity, we shall consider here only one of these eigendirections, as in [11]. The individual stem model is shown in Fig. 1a. It consists of a straight rod of height $h$ with a point mass $m$ at height $h_{b}$, which is the position of the center of gravity. When the stem is pulled away from its vertical equilibrium, it is subjected to two counteracting forces: one resulting from gravity, the other from stem elasticity. The linear mass-spring oscillator equation that describes the kinematics of an isolated stem is,

$$
m h_{b}^{2} \frac{d^{2} \theta}{d T^{2}}+C \frac{d \theta}{d T}+K_{T} \theta=0 .
$$

where $K_{T}=K-m g h_{b}$ is the apparent rotational stiffness of this oscillator, $K$ being the rotational stiffness due to stem elasticity, and $C$ a dissipation term.

In the simplest idealization, a canopy can be viewed as a row of stems along the main direction of wind gust propagation $[7,9]$. Thus, we introduce a space coordinate $X$ in the direction of wind. Let us consider a one-dimensional alignment of identical stems equally spaced by a distance $d$. The angular deflection $\theta$ is a discrete function of space, denoted $\theta_{j}=\theta\left(X_{j}\right)$. As neighboring stems may interact with each other, a spring of undeformed length $s$ and stiffness $r$ is added to the preceding model (see Fig. 1b). For small angles, the linearized torque of the force exerted by the stem number $j+1$ on the stem number $j$ is $M o_{j}=K_{I}\left(\theta_{j+1}-\theta_{j}\right)$. The dynamics of the oscillator number $j$ is thus coupled to that of its neighbors by,

$$
\begin{aligned}
& m h_{b}^{2} \frac{d^{2} \theta_{j}}{d T^{2}}+C \frac{d \theta_{j}}{d T}+K_{T} \theta_{j}-K_{I}\left[\left(\theta_{j-1}-\theta_{j}\right) F_{j}-\left(\theta_{j}-\theta_{j+1}\right) F_{j+1}\right] \\
& \quad=0 .
\end{aligned}
$$

where $F$ is the contact function defined by

$$
F_{j}=H\left[\theta_{j+1}-\theta_{j}+(d-s) / h\right],
$$

$\mathrm{H}$ being such that $H(x)=1$ for $x>0$ and $H=0$ otherwise (Heaviside function). The function $F$ expresses the geometric conditions for contact to occur between two adjacent oscillating stems. When there is no contact, the distance between the plants is larger than 


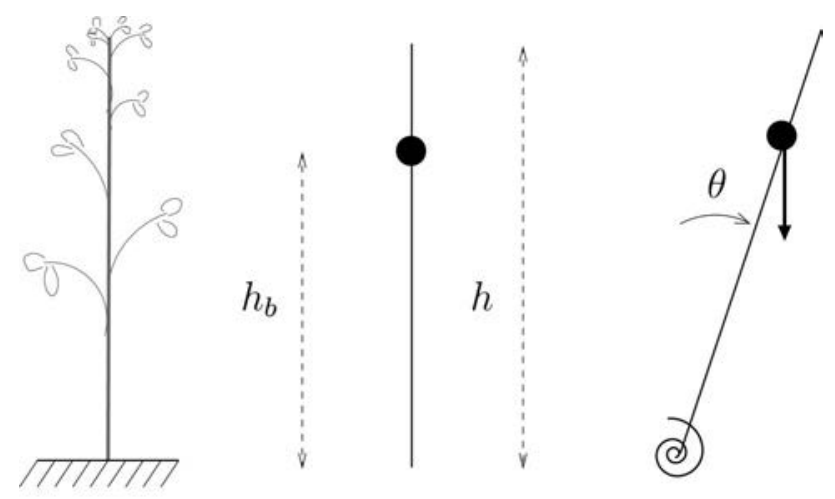

(a)

$S$

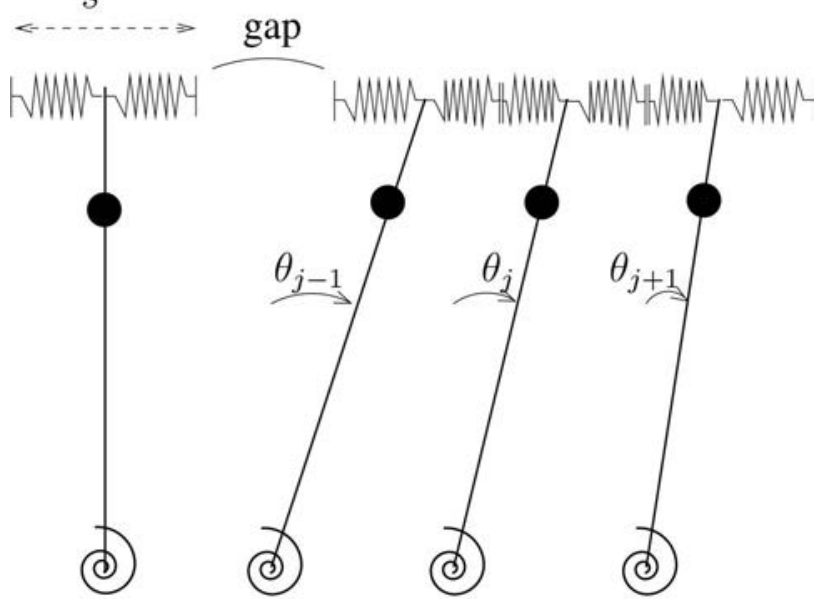

(b)

Fig. 1 (a) Schematic view of the stem model, a straight rod of height $h$, with a point mass at the height $h_{b}$, subjected to gravity and a restoring momentum from a torsional spring at the basis of the rod. (b) Schematic view of the stem interaction model. A spring is added on the rod, to simulate the foliage. Depending on the angles of two adjacent stems, they can be in contact or there can be a gap.

the gap, $F=0$. When a contact occurs and an elastic restoring force is exerted by stem number $j+1$ on stem number $j, F>0$.

2.2 Continuous Crop Canopy Model. Provided $d$ is small compared to $l_{\theta}=\theta /(\partial \theta / \partial X)$, the characteristic length of variation of $\theta$. We may express equation (2) in terms of a continuous function $\theta(X)$. The contact function in equation (3) then becomes,

$$
F(\theta)=H\left[d \frac{\partial \theta(X, T)}{\partial X}+\frac{d-s}{h}\right] .
$$

The fourth term of equation (2), i.e. the moment caused by contact between neighboring stems becomes,

$$
M_{I}=K_{I} d^{2} \frac{\partial^{2} \theta(X, T)}{\partial X^{2}} .
$$

We finally obtain the dynamic equation for the evolution of the angle $\theta$,

$$
m h_{b}^{2} \frac{\partial^{2} \theta}{\partial T^{2}}+C \frac{\partial \theta}{\partial T}+K_{T} \theta-F(\theta) K_{I} d^{2} \frac{\partial^{2} \theta}{\partial X^{2}}=0 .
$$

which has the form of a wave equation for an equivalent continuous medium.

2.3 Models of Forcing by Wind Loads. The wind over the crop canopy is liable to a shear-layer type instability that gener- ates sets of three to four transverse vortices or rollers with a typical wavelength ranging from three to seven times the height of the canopy, as modelled in [1]. These "trains of rollers" usually travel at a horizontal speed of approximatively 0.65 times the windspeed [12]. They produce intermittent gusts. When passing over a stem, the associated horizontal aerodynamic drag bends the stem. After this mechanical loading, the stem sways in free oscillations until a new vortex arrives, and a new gust induces a new wind-load. A simple model of this wind load induced by a travelling gust was suggested by Farquhar [9]. We also model the wind load as a force moving at a constant velocity $V$. Here the arerodynamic drag is zero for $X<V T$, which allows the stems to sway back after the gust. Thus, the moment to be added to the right hand side of equation (6) is

$$
M(X, T)=M_{0} H(X-V T) .
$$

To take into account the fact that vortices often travel in trains, we will also use a forcing function model involving a sinusoidal force on a crop canopy of infinite extent in the $X$ direction, displaced at the velocity $V$,

$$
M(X, T)=M_{0} \sin [\alpha(X-V T)],
$$

where $\alpha$ corresponds to the wavenumber of the forcing function, and also scales the forcing frequency experienced by an individual stem. 
These two models of forcing by wind, although simple, allow us to study two main characteristics of the crop canopy, free oscillations after a single wind gust and resonant response. See [4] for the relation between the magnitude $M_{0}$ and the aerodynamical characteristics of the wind and of the stem foliage in the case of wheat.

2.4 Non-Dimensional Model. Let us introduce the following non-dimensional parameters, $\lambda=(d-s) / d, D=d / h$ and $b$ $=h_{b} / h$. Let $\omega_{T}=\sqrt{K_{T} / m h_{b}^{2}}, \omega_{I}=\sqrt{K_{I} / m h_{b}^{2}}$ and $\omega_{C}=C / m h_{b}^{2}$ be the characteristic frequencies based on the stems stiffness, elastic interaction stiffess and stem damping respectively. The nondimensional space and time variables are $x=X / d$ and $t=T \omega_{T}$. We may also define the following non-dimensional frequencies and torque,

$$
\gamma=\frac{\omega_{C}}{\omega_{T}}, \quad \kappa=\frac{\omega_{I}}{\omega_{T}}, \quad \mu=\frac{M}{m h_{b}^{2} \omega_{T}^{2}} .
$$

With these terms, the wave equation for the canopy motion becomes,

$$
\frac{\partial^{2} \theta}{\partial t^{2}}+\gamma \frac{\partial \theta}{\partial t}+\theta-f_{c} \kappa^{2} \frac{\partial^{2} \theta}{\partial x^{2}}=\mu(x, t),
$$

where the contact function is,

$$
f_{c}(\theta)=H\left[\frac{\partial \theta(x, t)}{\partial x}+\frac{\lambda d}{h}\right] .
$$

All the forcing functions considered previously can be written in the form of $\mu(x, t)=\mu(x-v t)$, that is as a waveform of constant shape moving at the dimensionless velocity $v=V / d \omega_{T}$. Assuming that $\theta$ is also a function of $x-v t$, we introduce the change of variables $\xi=x-v t$, which yields

$$
\left(v^{2}-f_{c} \kappa^{2}\right) \theta^{\prime \prime}-\gamma v \theta^{\prime}+\theta=\mu(\xi),
$$

where ()$^{\prime}$ indicates differentiation with respect to $\xi$. In equation (12), all parameters can be determined by experiments involving one or two stems. These experiments are described in the following section, where typical values of each parameter are estimated.

The wave equation (10) and equation (12) are simple equations in which the only nonlinearity is due to the Heaviside function, which represents the conditional contact between neighboring stems.

\section{Measuring Model Parameters in Alfalfa}

3.1 Experimental Conditions. Plants of alfalfa (Medicago sativa L. cv Mercedes) were grown from cuttings in individual pots filled with a mixture of local clay soil, peat moss and sand. All the plants were grown in a climate controlled glass-house at the INRA research center in Lusignan (France), with standard agricultural practices. The pots were arranged to mimic a minisward with a density of 50 plants per meter squared. When the dominant stems of the plants reached a height of approximately $0.45 \mathrm{~m}$, individual plants were thinned to a single stem per pot and mechanical measurements were taken.

3.2 Mass-Spring Oscillator Parameters. The experimental set-up for measuring the mass-spring parameters is based on free oscillation tests as decribed by Flesh [11]. The kinematics of stem motion was recorded using a video camera. Each individual stem was characterized using the following process. First of all, a direction in which the movement remained within a plane was determined. The stem was then placed in front of a digital videocamera such that the plane of movement is perpendicular to the camera axis. The stem was displaced from its static equilibirum position by hand and released, and its motion recorded. A typical image sequence is represented on Fig. 2. Only the green channel of the original RGB image is represented. To track the motion of

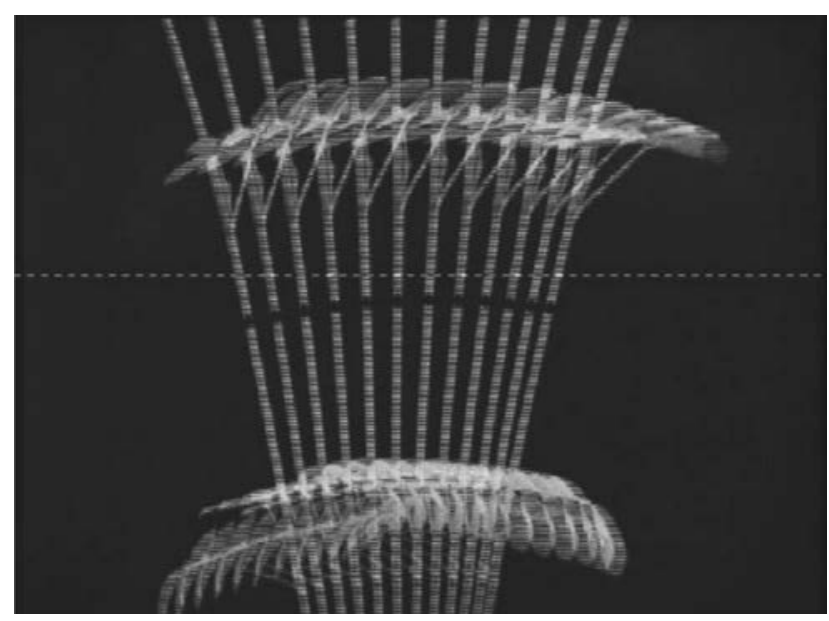

Fig. 2 Image sequence showing snapshots of the stem position. The dashed line indicates the height of which the maximum value of the green channel was searched. Only the green channel of the original RGB image is shown.

the stem, a single horizontal line of the film is isolated (white line of Fig. 2). The position of the pixel at which the value of the green channel is maximum gives the $x$ position of the stem at the chosen height. Thus the position of the stem as a function of time was recorded. The corresponding evolution of the angular position for a typical experiment is plotted versus time in Fig. 3. The good agreement between experimental data and the behavior predicted by the model, equation (1) indicates that the mass-spring oscillator approximation is sufficient to describe the dynamical behavior of an isolated stem.

To estimate the modal mass $m$ and the altitude of the center of gravity $h_{b}$, each stem was then cut into four equal parts, and each part was weighted immediately (so that changes in weight due to drying were negligible). The modal mass was calculated according to $m=\int_{0}^{h} m(x) \Phi^{2}(x) d x$, where $\Phi(x)$ is the modal shape of the stem movement. In our model, $\Phi(x)=x$. Figures 4 and 5 display the measured values of $m, h_{b}, \omega_{T}$, and $\gamma$ as functions of $h$. Simple biometric relations, relating $h_{b}$ and $m$ to the overall height of the stem were established, $h_{b}=0.65 h$ and $m=8.5$ $\times 10^{-3}\left(\mathrm{Kg} \cdot \mathrm{m}^{-3}\right) h^{3}$, see Fig. 4. Figure 5 shows that $\omega_{T}$ and $\gamma$ do not vary significantly with $h$. We use $\omega_{T}=3.4 \mathrm{~Hz}$ and $\gamma=0.34$.

3.3 Elastic Interaction Parameters. A second set of experiments was designed to characterize the elastic interaction between stems. Five sets of two stems taken from the plants used in the free motion experiments were selected. The two stems were

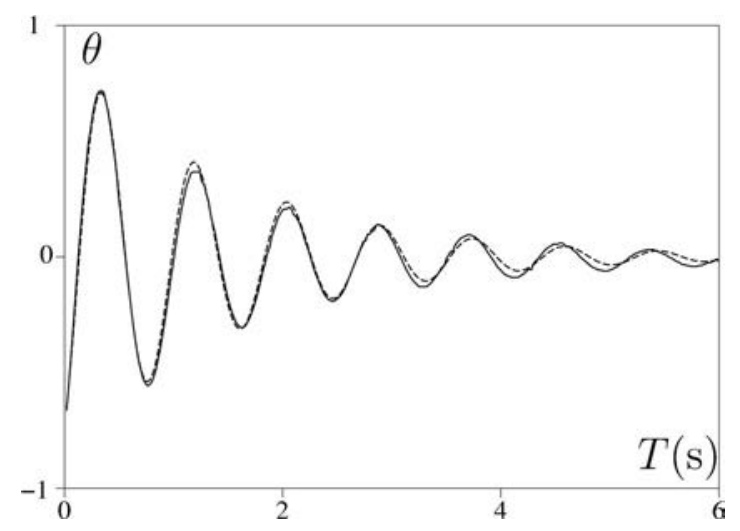

Fig. 3 Stem angular position as a function of time, experiment (solid line) and theory (dashed line) 


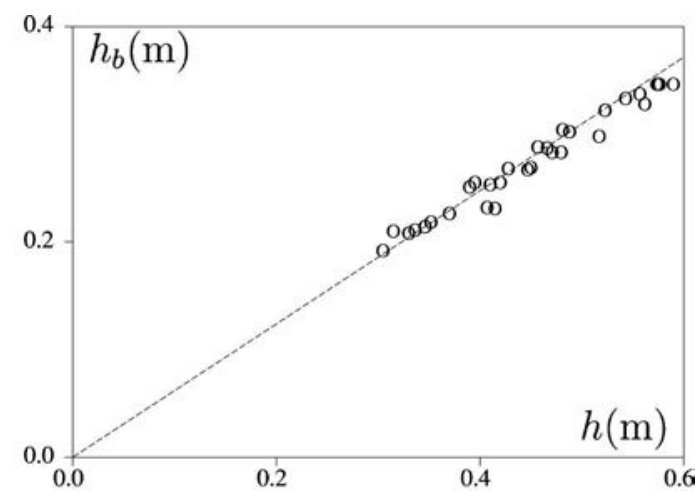

(a)

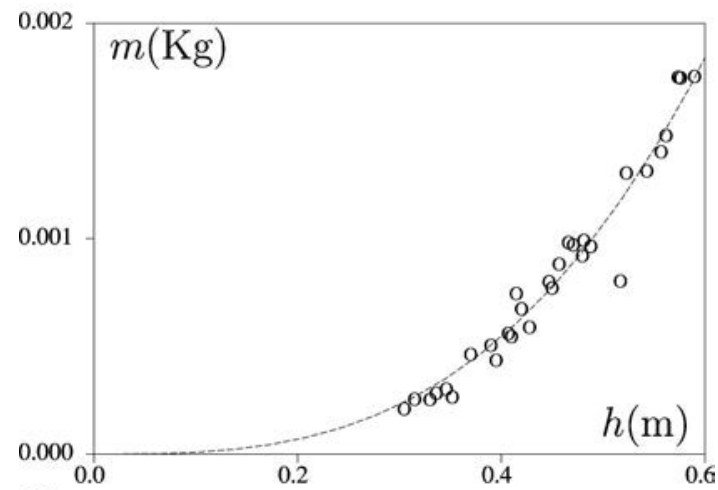

(b)

Fig. 4 Biometric properties that vary with plant height (a), height of the center of gravity $h_{b}$ as a function of the stem height $\boldsymbol{h}$; $(\bigcirc)$, experiments; $(---)$, linear approximation $\boldsymbol{h}_{\boldsymbol{b}}$ $=0.65 h ;(b)$, modal mass $\boldsymbol{m}$ as a function of the stem height $\boldsymbol{h}$; (O), experiments; $(---)$, cubic approximation $m=8.5$ $\times 10^{-3}\left(\mathrm{Kg} \cdot \mathrm{m}^{-3}\right) h^{3}$

placed so that their eigenplanes of oscillation coincided and their leaves were just in contact (Fig. 6). One of the stems was then bent away form its static equilibrium position and then released as in the previous section. At some point, it impinged the second stem, so that both started oscillating. The movement of the two stems (i.e., their positions over time) was recorded using the same setup as in the previous section. The positions of stems $\theta_{1}$ and $\theta_{2}$ during a typical experiment are presented in Fig. 7. When $\theta_{2}$ $<\theta_{1}$ the stems are in contact and the elastic restoring force acts. The two leaves act as springs. The duration of contact $T_{c}$ is also represented.

When in contact, the evolution of the two stems are described by two coupled linear equations derived from equation (2),

$$
\begin{aligned}
& m_{1} h_{b 1}^{2}\left(\ddot{\theta}_{1}+\gamma \omega_{T} \dot{\theta}_{1}+\omega_{T}^{2} \theta_{1}\right)=K_{I}\left(\theta_{2}-\theta_{1}\right), \\
& m_{2} h_{b 2}^{2}\left(\ddot{\theta}_{2}+\gamma \omega_{T} \dot{\theta}_{2}+\omega_{T}^{2} \theta_{2}\right)=K_{I}\left(\theta_{1}-\theta_{2}\right) .
\end{aligned}
$$

All parameters in these equations are known from the single-stem experiments described in the previous section, except $K_{I}$. By numerically integrating equation (13), the relation between $K_{I}$ and the duration of contact may be derived. This allows $K_{I}$ to be adjusted to the experimental data of $T_{c}$. The initial conditions at contact $\theta_{1}=\theta_{2}=\dot{\theta}_{1}=0$ and $\dot{\theta}_{2}$ are deduced from the data plotted in Fig. 7. The value of $K_{I}$ is the only unknown. Hence, its value may be numerically derived from the duration of contact. The values of $K_{I}$ for each set of stems give us the mean value $K_{I}$ $=6.2 \pm 3 \times 10^{-3} \mathrm{~N} \cdot \mathrm{m}$.

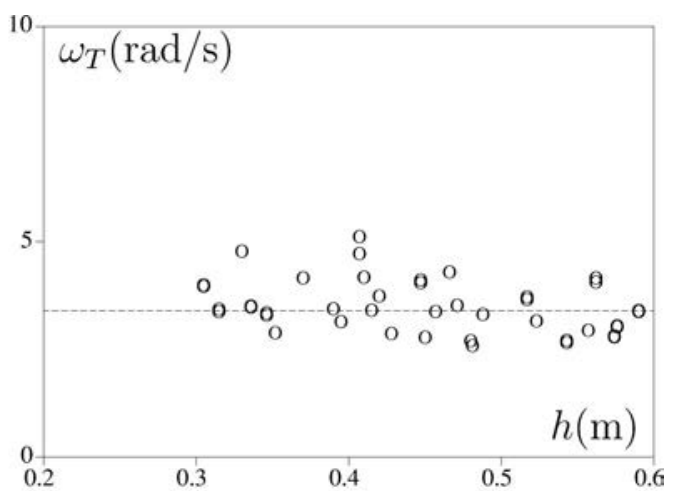

(a)

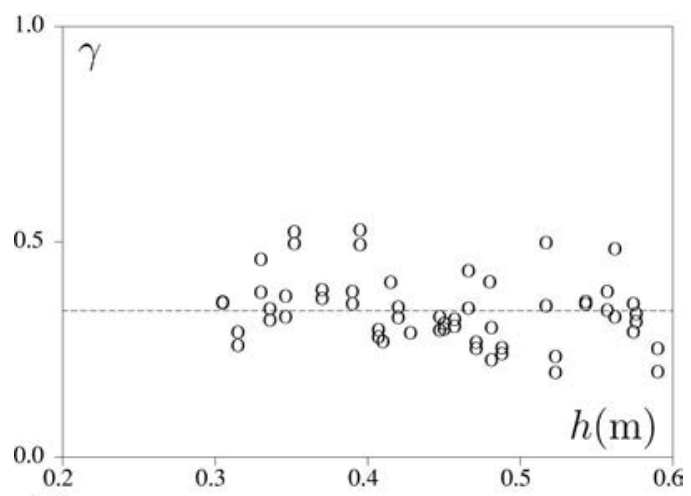

(b)

Fig. 5 Dynamic properties, (a), frequency $\omega_{T}$ as a function of the stem height $\boldsymbol{h} ;(\bigcirc)$, experiments; $(---)$, approximation by $\omega_{T}=3.4 \mathrm{~Hz}$; $(b)$, non-dimensional dissipation $\gamma$ as a function of the stem height $\boldsymbol{h}$; $(\bigcirc)$, experiments; $(---)$, approximation by $\gamma=0.34$

\section{Simulation of Stem Motions in an Alfalfa Canopy Submitted to Wind}

Let us first consider the model defined in section 2, equation (12), using the parameters of the experiments of section 3 for a

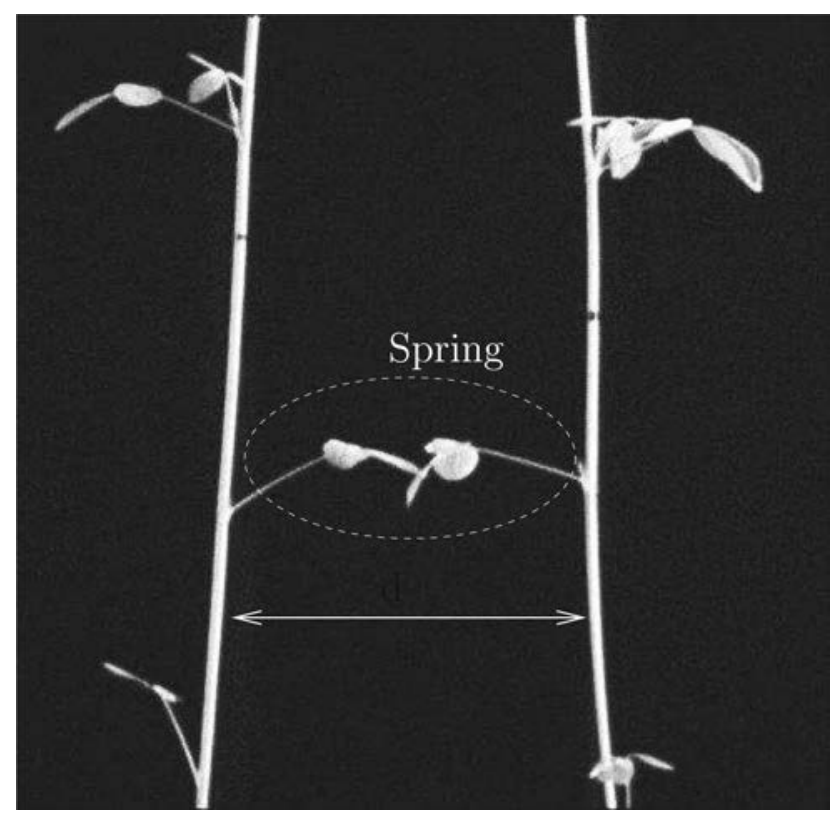

Fig. 6 Snapshot of a contact between leaves 


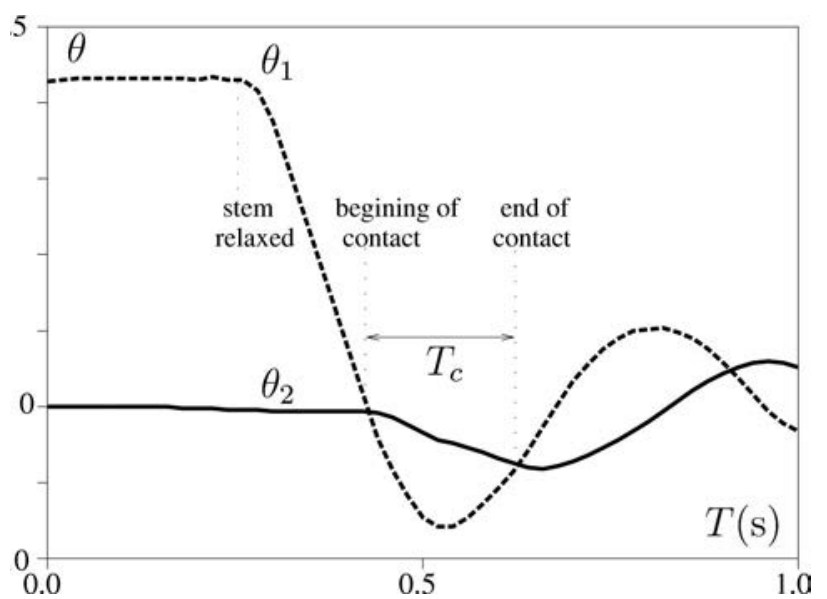

Fig. 7 Representative evolution of stem positions in a contact experiment as function of time. Contact occurs over a period of duration duration $T_{c}$.

typical uniform alfalfa crop of height $h=0.4 \mathrm{~m}$, with individual stems of size $s=0.045 \mathrm{~m}$ equally spaced by a distance $d$ $=0.05 \mathrm{~m}$. We also recall the experimental values of $\gamma=0.34$ and $K_{I}=6.2 \times 10^{-3} \mathrm{~N} \cdot \mathrm{m}$, which for the selected value of $h$ correspond to $\kappa=3.8$. We choose $V=1 \mathrm{~m} / \mathrm{s}$, hence $v=5.88$.

4.1 Response to a Single Wind-Pulse. The forcing model of a gust of wind, that moves at the velocity $V$ was introduced in equation (7). We have $\mu(\xi)=\mu_{0} H(\xi)$ and we are interested in the oscillations of the crop for $\xi<0$, behind the gust. We set $\mu$ equal to 0.6 in order to observe significant nonlinear effects. We solved equation (12) for $\xi<0$, with initial conditions at $\xi=0$ using the static response of the crop, $\theta_{0}=\mu_{0}$ and $\theta_{0}^{\prime}=0$. The numerical solutions are plotted in Fig. 8. In Fig. 8a, no stem interaction was considered, as in [9]. In Fig. 8b, elastic stem interactions were considered. The angular deformation $\theta$ as well as the shape of the crop canopy are plotted as function of $X$ behind the burst of wind. The elastic restoring force tends to increase the slope of the $\theta$ curve. This is associated to the local increase of stiffness due to contact.

4.2 Response to Infinite Length Sinusoidal Wave Train. We will now consider the sinusoidal forcing function $\mu(\xi)$ $=\mu_{0} \sin (\alpha \xi)$ for the wind load. The magnitude of $\mu$ was chosen equal to 0.3 , which is 0.6 peak to peak as above. Figure 9 shows the effect of the wavenumber $\alpha$ on the amplitude of motion in two cases: (a) model with no interaction, (b) model with interaction. Case (a) is a simple linear problem for which the expression of the transfer function between force and response is,

$$
\frac{A}{\mu_{0}}=\frac{1}{-v^{2} \omega^{2}-i \gamma v \omega+1},
$$

and has a maximum at $\alpha=1 / v=0.17$. Case (b) was solved numerically. The maximum amplitude is larger and occurs at a lower wave number in case (a) than in case (b). If contact was permanent between stems $\left(f_{c}=1\right)$, the transfer function would be,

$$
\frac{A}{\mu_{0}}=\frac{1}{\left(\kappa^{2}-v^{2}\right) \omega^{2}-i \gamma v \omega+1},
$$

which would have a maximum at $\alpha=\left(v^{2}-\kappa^{2}\right)^{-1 / 2}=0.22$. The case (b) lies between the two linear cases.

\section{Discussion}

Numerous collisions between shoots can be observed in a canopy exposed to turbulent wind loads. However the effects of

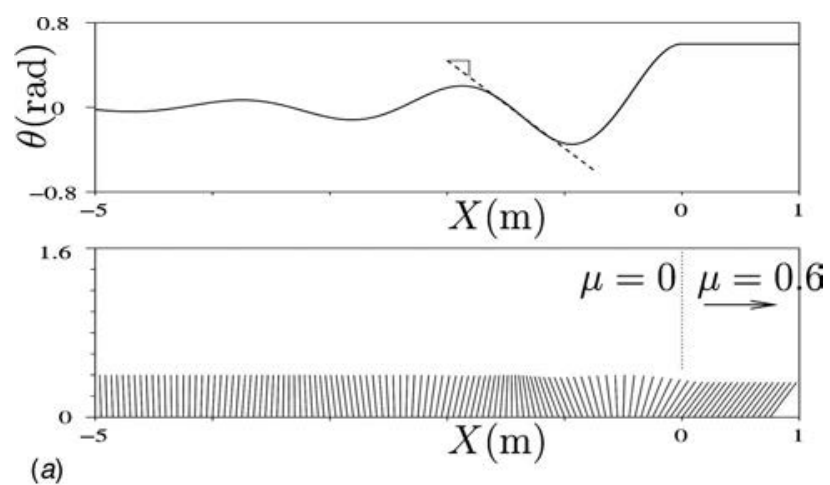

(a)

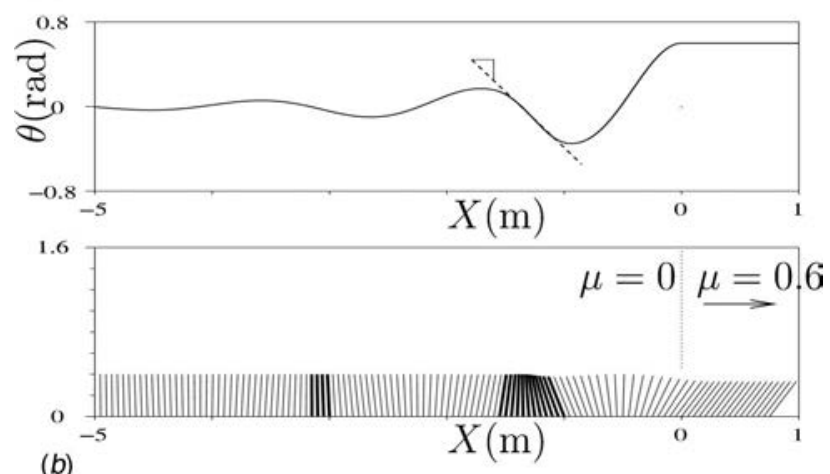

Fig. 8 Instantaneous shape of the field behind the wind gust. For $X>0$ the wind is uniform $\left(\mu_{0}=0.6\right)$ and for $X<0$ there is no wind $\left(\mu_{0}=0\right)$. The variation of $\theta$ as function of $X$ is plotted and the crop canopy for $h=0.4 \mathrm{~m}$ and $d=0.05 \mathrm{~m}$ is sketched; (a), no interaction between stems, $\kappa=0 ;(b)$, interactions between stems, $\kappa=3.8$; stems in contact are in bold.

such collisions on the global behavior of a canopy have often been overlooked. In this paper, a model equation was proposed for the elastic collisions between pairs of neighboring shoots. The two shoots are considered as two 1-D oscillators and only the nonlinearity due to the loss of contact was taken into account. This effect was included into the discrete model of crop canopy exposed to wind loads suggested in [9]. Moreover, it was shown that this enhanced discrete model including stems oscillations and shootto-shoot elastic collisions could be expressed as a wave equation for an equivalent continuous medium.

A novel experimental method based on video recording of stem movements and simple image analysis was implemented to quantify the different parameters of the enhanced discrete model and of the continuous wave equation. This video tracking is a noncontact method. As plant stems are very lightweight and flexible structures, a non-contact method has less design constraints and possible artifacts than the previous measurements using angular sensors [11] or strain gauges [2]. It is also much faster, so that more plants can be studied. The analysis of shoot-to-shoot collisions conducted with this method revealed that our model equation for elastic collisions could describe the results of collisions between pairs of shoots in alfalfa reasonably well.

Numerical simulations using alfalfa parameters demonstrated that the effect of elastic collisions between neighboring plants had significant effects on the dynamical behavior of the model canopy, when exposed to a single gust of wind or to a train of wind gusts. In particular the dynamics of free oscillations behind the wind load became non linear and the frequency spectrum of the canopy in response to periodic forcing was modified. The resonant eigenfrequency of the canopy including elastic contact was found to be almost 20\% higher, compared to a non-contact model. These changes may have an indirect influence on thigmomorphogenesis or $\mathrm{CO}_{2}$ vertical convection within the canopy [7]. It is also likely 


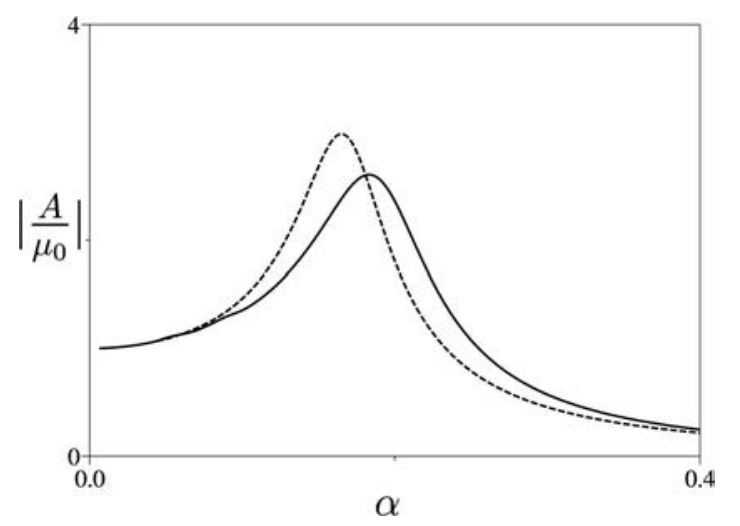

Fig. 9 Transfer function between force and response as function of the wavenumber $\alpha$ for $\kappa=3.8, v=5.88, \mu_{0}=0.3 ;(-), f_{c}$ defined by equation (11); $(---), f_{c}=0$

to influence stem lodging. Indeed, due to finite anchorage strength, it is usually considered that a minimum offset is required between resonant and forcing frequency to provide safety against lodging [13]. In wheat, for example, it has been shown that the resonant frequency of individual stalk vibration at ear maturity (a critical stage for lodging in wheat) was slightly above the broad shoulder of typical wind frequency spectra [13]. Contacts between plants are thus likely to enhance this security factor, in addition to provide additional support to plants.

It should also be noted that the simulations presented here were conducted using a density that reflected the conditions of an experimental mini-sward in which the stem density was adjusted so that neighbor shoots only have definite contacts. In natural conditions however the shoot densities are much higher. Gosse [14] reported shoot densities on rows ranging from 100 to 150 stems $\cdot \mathrm{m}^{-1}$. Therefore, the mean distance between stems should lie between 0.5 to $1 \mathrm{~cm}$, that is 5 to 10 times less than in our conditions. Contacts and collisions are thus expected to have even higher effects in "real" crops than the one observed in our conditions. Note that such conclusion is not limited to alfalfa. Contacts between plants can be observed in most crops (like wheat, corn...). As our approach is not dependant on the species, it should thus proved useful in assessing the effects of elastic contacts in crops.

In future, three main improvements could be considered. First, the existing models for the dynamics of individual plants should be considered. For instance mode shapes other than linear shape, finite rotation effects and out of plane motion of plants could be considered. When considering a crop canopy model, the last effect would require a 2-D population of plants, instead of a single row.

Second, it would be interesting to analyze crop motion using more complex and realistic wind forcing models [1]. The continuous model of crop motion presented here should prove a very useful tool for studies of the interactions between wind flow and canopy motion. Furthermore the simulation time is significantly smaller than with discrete models because the discretisation in space is not related to the actual distance between stems. Moreover the wave equation can be studied analytically.

Third, other types of interactions between stems could be considered. Dissipation resulting from collisions and solid frictions between plants are likely to be very significant, especially in very dense crop canopies. The quantification of these effects (as well as possible validations of the models) requires the motions of sets of plants in real canopies to be measured directly. Finally, betweenplant variations and their effect could also be considered. It is worth mentioning that our methodology is directly applicable to the study of such inhomogeneities, yielding a new equation with probabilistic values of the parameters. These aspects are currently under investigation.

\section{References}

[1] Finnigan, J., 2000, “Turbulence in Plant Canopies," Annu. Rev. Fluid Mech., 32, 519-571

[2] Finnigan, J., 1979, “Turbulence in Waving Wheat," Boundary-Layer Meteorol., 16, 181-211.

[3] Flesh, T. K., and Grant, R. H., 1991, "The Translation of Turbulent Wind Energy to Individual Corn Plant Motion During Senescence," Boundary-Layer Meteorol., 55, 161-177.

[4] Farquhar, T., Wood, J. Z., and van Beem, J., 2000, "The Kinematics of Whea Struck by a Wind Gust," ASME J. Appl. Mech., 67, 496-502.

[5] Jaffe, M. J., and Forbes, S., 1993, "Thigmomorphogenesis: The Effect of Mechanical Perturbation on Plants," Plant Growth Regulation, 12(3), 313324.

[6] Coutand, C., and Moulia, B., 2000, "A Biomechanical Study of the Effect of a Controlled Bending on Tomato Stem Elongation: II Local Mechanical Analysis and Spatial Integration of the Mechanosensing," J. Exp. Bot., 51(352), 18251842.

[7] Farquhar, T., and Eggleton, C. D., 2000, "Pulsatile Flow Heightens Vertical Exchanges in a Wheat Canopy," in Proceedings of the 3rd Plant Biomechanics Conference, Freiburg, Spatz, H. C. and Speck, Th. Eds.

[8] Niklas, K. J., and Speck, T., 2001, "Evolutionary Trends in Safety Factors Against Wind-Induced Stem Failure," Am. J. Bot., 88, 1266-1278.

[9] Farquhar, T., Zhou, J., and Haslach, H., 2003, "A Possible Mechanism for Sensing Crop Canopy Ventilation," Ch. 15, in Sensors and sensing in biology and engineering, ed. FG Barth, JAC Humprey, T Secomb, Springer Press, Wien/New York.

[10] Flesch, T. K., and Grant, R. H., 1992a, "Corn Motion in the Wind During Senescence: I Motion Characteristics,” Agronomy Journal, 84, 742-747.

[11] Flesh, T. K., and Grant, R. H., 1992b, "Corn Motion in the Wind During Senescence: II Effect of Dynamic Plant Characteristics," Agronomy Journal, 84, 742-747.

[12] Raupach, M. R., Finnigan, J. J., and Brunet, Y., 1996, "Coherent Eddies and Turbulence in Vegetation Canopies: The Mixing-Layer Analogy," BoundaryLayer Meteorol., 78, 351-382.

[13] Farquhar, T., and Meyer-Philips, H., 2001, "Relative Safety Factors Against Global Buckling, Anchorage Rotation, and Tissue Rupture in Wheat," J. Theor. Biol., 211, 55-65.

[14] Gosse, G., Lemaire, G., Chartier, M., and Balfourier, F., 1988, "Structure of a Lucerne Population (Medicago Sativa L) and Dynamics of Stem Competition for Light During Regrowth," J. Appl. Ecol., 25, 609-617. 